\title{
Magnetohydrodynamic Flow Through Ducts with Different Cross Section
}

\author{
M.Prasanna Jeyanthi, S.Ganesh
}

\begin{abstract}
In this article the coupled convection diffusion equations of Magneto- hydrodynamic flow problem have been solved. The solution of the problem is presented for various cross sections like square, rectangle, triangle, circle, ellipse, sector and annulus under steady state conditions. Ducts which are electrically insulated walls have been considered in this study. Some contours are presented to show the behaviors of the magnetic field and velocity.
\end{abstract}

Keywords: Magnetohydrodynamic (MHD) flow, Finite Difference Method, Insulated walls, Polygonal Duct, Circular Duct

\section{INTRODUCTION}

The investigation of magnetic properties of the electrically conducting fluid (for example, metal solution, salt water, electrolytes, plasma) is Magnetohydrodynamics(MHD). MHD pipe flow through duct often occur in Nuclear reactors, measurements of blood flow MHD pumps and generators. Researchers have made lot of contributions in applications of MHD flow through ducts. Initially, Hartmann.J and Lazarus.F [13] studied MHD flow between two plates that are parallel with electrically conducting fluid. Then J.A. Shercliff ,R.R.Gold, C.Chang et.al [8, 12, 14] and many authors discussed about MHD flow problems with conducting and nonconducting walls. Only for certain regular geometric regions analytical solutions exist. . Analytical solution of rectangular pipe with insulated walls have been presented by J.A. Shercliff [20]. Numerical solution of the MHD problem have been obtained using Chebychev method (Ibrahim) [9], Differential Quadrature method (M.Tezer-Sezgin) [22], Finite difference method (Muchim Chutia) [10], Finite element method(A.I.Nesliturk et. al and Hunt) [1, 18], Boundary Element method(M.Tezer-Sezgin et.al), $[19,21]$ and Radial basis function generated finite difference method (M.Prasanna Jeyanthi) [17]. Hunt [16] and C.C.Chang et.al presented the solution for rectangular duct with the effect of conducting walls. C.Bozkaya et.al [7] used Boundary element method to solve the MHD flow problem with partially conducting walls and rectangular duct with non conducting walls under the given magnetic field through an angle. MHD pipe flow with triangular cross section have been solved numerically in [2], [4], [5], [6]. Shercliff [15] derived the

Revised Manuscript Received on October 22, 2019.

* M.Prasanna Jeyanthi

M.Prasanna Jeyanthi*, Department of Mathematics, Sathyabama Institute of Science and Technology, Chennai, India. Email: prasannajeyanthi@gmail.com

Dr.S.Ganesh, Department of Mathematics, Sathyabama Institute of Science and Technology, Chennai, India. Email: solution of MHD flow problem with arbitrary cross section in presence of uniform magnetic field. Shercliff compared his solution [15] with his experimental results in [14]. R.R.Gold [12] presented the analytical solution of MHD pipe flow with circular cross section and com- pared the results with the experimental data given by Shercliff [14]. S.Han Aydin presented the solution of MHD channel flow with circle as cross section by using BEM and FEM-BEM. M.Tezer Sezgin and S.Han Aydin analyzed the solution of MHD pipe flow for electrically conducting, incompressible and viscous fluid through z-direction which has rectangular, circular and elliptical cross section in the xy-plane. The analytical solution of the electrically conducting fluid through the duct with circular sector as cross section under uniform magnetic field have been derived using Green function method [23].

In most of the papers, the discussions are made for rectangular, triangular and circular ducts. All the well known geometric shapes (Square, rectangle, triangle, pentagon, hexagon, circle, ellipse, circular annulus, circular sector) of channels have been included in this paper for discussion with the fixed Hartmann number $M=10$. In most of the papers, the discussions are made for rectangle triangle and circle. The characteristics of MHD flow through ducts with cross sections of different shapes have been studied this paper.

\section{FORMULATION OF THE PROBLEM}

The fluid considered in this problem is electrically conducting which flows through the pipe with uniform cross section. The applied magnetic field makes an angle with $\mathrm{y}$-axis. The assumption made in the problem are

1. The walls of the ducts being electrically insulated.

2. The conductivity of the fluid is being finite.

3. All the properties of the fluid are constant except pressure gradient

4. There is no variation in duct with uniform the cross section and no secondary flow.

It is assumed that the fluid moves through $\mathrm{z}$ - axis where as xy-axis is the axis of cross section. The flow and the direction of the fluid is bolstered by constant pressure gradient $\frac{\partial p}{\partial x}$.

Let $\theta$ be the angle made by the applied uniform magnetic field with the y-axis. In steady state the MHD flow through ducts are governed by the equations, 


$$
\begin{aligned}
& \nabla \cdot \vec{V}=0 \\
& \nabla . \vec{B}=0 \\
& \quad \operatorname{CurlH}=4 \pi \vec{j} \\
& j=\sigma(\vec{E}+\mu \vec{V} \times \vec{B}) \\
& \nabla \times \vec{j}=\sigma(\nabla \times \vec{E}+\nabla \times(\mu \vec{V} \times \vec{B}) \\
& \nabla \times \vec{B}=4 \pi \vec{j}
\end{aligned}
$$

where $\dddot{j}$ is the electric current density, $\rho$ is fluid density, $\vec{E}$ is electric field, $\mu$ is the permeability , B is magnetic field, and $\mathrm{V}$ is fluid velocity. Equations (1) to (6) can be written in the non dimensional form of coupled system of equations in magnetic field and velocity [11].

$$
\begin{array}{ll}
\nabla^{2} V+M_{x} \frac{\partial B}{\partial x}+M_{y} \frac{\partial B}{\partial y}=-1 & \text { in } \Omega \\
\nabla^{2} B+M_{x} \frac{\partial V}{\partial x}+M_{y} \frac{\partial V}{\partial y}=0 & \text { in } \Omega
\end{array}
$$

where $B(x, y), V(x, y)$ instigate the magnetic field and velocity respectively, $\Omega$ is the region of the duct, $\partial \Omega$ is the boundary of $\Omega, M_{x}=M \sin \theta, M_{y}=M \cos \theta$, Hartmann number $M$ is the norm of $\left(M_{x}, M_{y}\right)$. The boundary conditions are $B=0$ and $V=0$.

When $\theta=\pi / 2$ equations (7), (8) takes the form

$$
\begin{aligned}
& \nabla^{2} V+M \frac{\partial B}{\partial x}=-1 \quad \text { in } \Omega \\
& \nabla^{2} B+M \frac{\partial V}{\partial x}=0 \quad \text { in } \Omega
\end{aligned}
$$

\section{FINITE DIFFERENCE METHOD}

To solve the non dimensional coupled partial differential equations, Finite Difference method is used. Equations (7), (8) are decoupled using the substitution

$$
X=V+B, \quad Y=V-B
$$

Then equation (7), (8)_are of the form

$$
\begin{array}{ll}
\nabla^{2} X+M_{x} \frac{\partial X}{\partial x}+M_{y} \frac{\partial X}{\partial y}=-1 & \text { in } \Omega \\
\nabla^{2} Y+M_{x} \frac{\partial Y}{\partial x}+M_{y} \frac{\partial Y}{\partial y}=0 & \text { in } \Omega
\end{array}
$$

with the boundary conditions

$$
X=Y=0 \quad \text { on } \quad \partial \Omega
$$

The domain of the problem is partitioned into uniform grids. Equation (7), (8) can be written using finite difference method as follows.

$$
\begin{aligned}
& c_{4} X(j+1, k)+c_{5} X(j, k)=-1 \\
& d_{1} Y(j, k-1)+d_{2} Y(j, k+1)+d_{3} Y(j-1, k) \\
& +d_{4} Y(j+1, k)+d_{5} Y(j, k)=-1
\end{aligned}
$$

belong to the engineering and technology area. In the paper with the boundary conditions

$X(-1, k)=0=X(1, k)$ and

$X(j,-1)=0=X(j, 1)$

$Y(-1, k)=0=Y(1, k)$ and

$Y(j,-1)=0=Y(j, 1)$

where $n_{1}, n_{2}$ are the number of grid points in the $x$-axis and $y$-axis,

$c_{1}=\left(\frac{1}{k^{2}}-\frac{M_{y}}{2 k}\right), c_{2}=\left(\frac{1}{k^{2}}+\frac{M_{y}}{2 k}\right), \quad c_{3}=\left(\frac{1}{h^{2}}-\frac{M_{x}}{2 h}\right)$,

$c_{4}=\left(\frac{1}{h^{2}}+\frac{M_{x}}{2 h}\right), \quad c_{5}=\left(\frac{-2}{h^{2}}+\frac{-2}{k^{2}}\right), \quad d_{1}=\left(\frac{1}{k^{2}}+\frac{M_{y}}{2 k}\right)$,

$d_{2}=\left(\frac{1}{k^{2}}-\frac{M_{y}}{2 k}\right), \quad d_{3}=\left(\frac{1}{h^{2}}+\frac{M_{x}}{2 h}\right), \quad d_{4}=\left(\frac{1}{h^{2}}-\frac{M_{x}}{2 h}\right)$,

$d_{5}=\left(\frac{-2}{h^{2}}+\frac{-2}{k^{2}}\right), h=\frac{1}{n_{1}}$ and $k=\frac{1}{n_{2}}$.

Then the solutions of the equations (7), (8) can be found by using the equations (11).

\section{Polygonal Duct}

\section{A. SQuARE DuCT}

The centre of the square duct is positioned at the origin. The walls of the ducts are defined by $x= \pm 1, y= \pm 1$. The solutions of the equations (7), (8) are presented in contours for $\theta=\frac{\pi}{2}, \frac{\pi}{3}, \frac{\pi}{4} \frac{\pi}{6}$ for the fixed Hartmann number $\mathrm{M}=10$, with Dirichlet boundary condition.

$V=0, B=0$ for $x=-1$, and $-1 \leq y \leq 1$

$V=0, B=0$, for $x=1$, and $-1 \leq y \leq 1$

$V=0, B=0$, for $y=-1$, and $-1 \leq x \leq 1$

$V=0, B=0$, for $y=1, \quad$ and $-1 \leq x \leq 1$

The induced magnetic field and velocity contours for different angles $\theta=\frac{\pi}{2}, \frac{\pi}{3}, \frac{\pi}{4}$ are presented in

Figure $(1,2,3)$. 


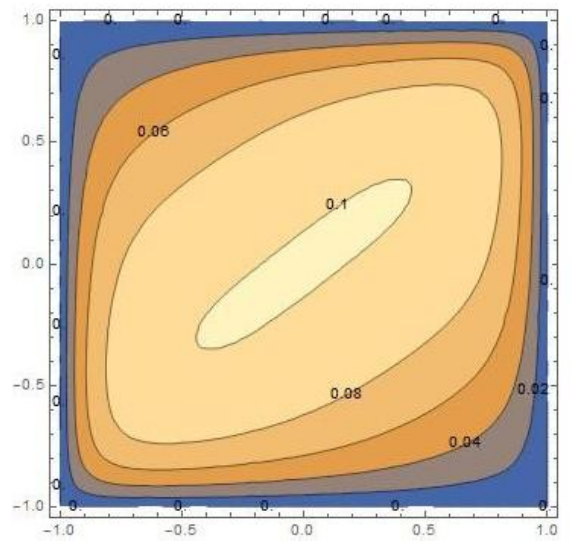

(a)

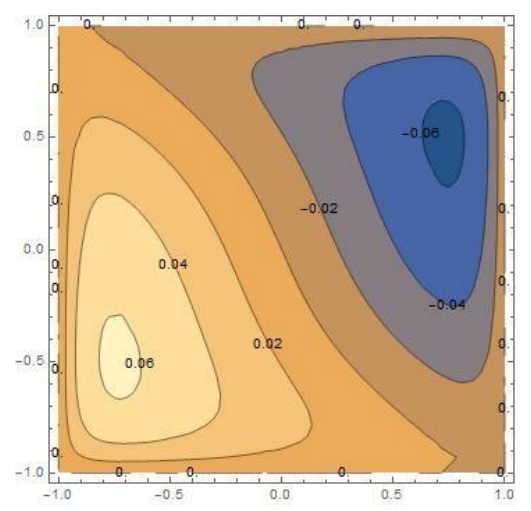

(b)

Fig.1. Square duct with $\theta=\pi / 3$ (a) Velocity (b) Magnetic Field

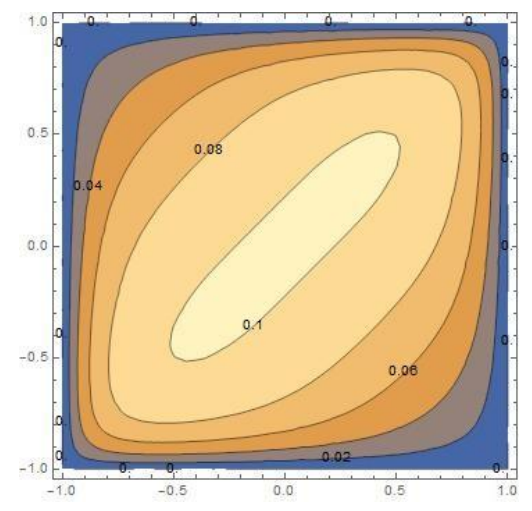

(a)

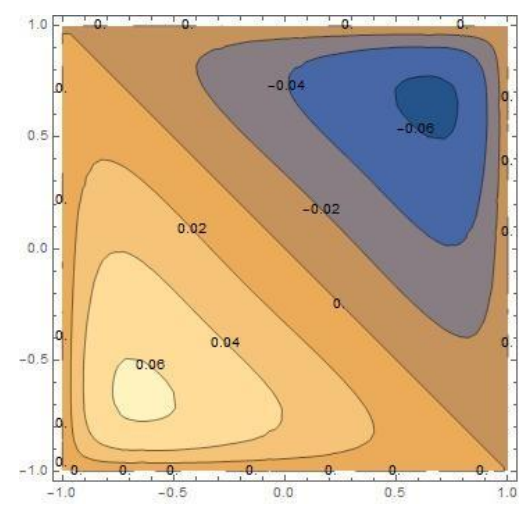

(b)

Fig.2. Square duct with $\theta=\pi / 4$ (a) Velocity (b) Magnetic Field

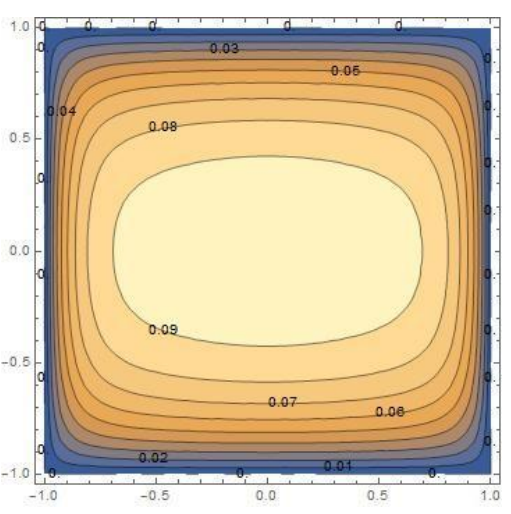

(a)

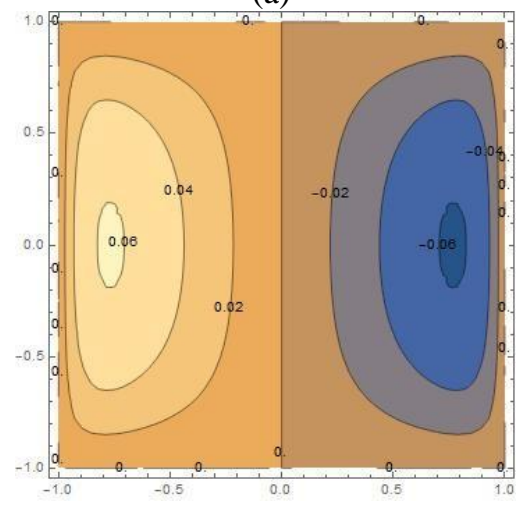

(b)

Fig.3.Square duct with $\theta=\pi / 2$ (a) Velocity (b) Magnetic 3

Field

\section{B. Rectangular Duct}

The insulated boundary of the rectangular duct are $0 \leq \mathrm{x} \leq 1$ and $-1 \leq y \leq 1$. The induced magnetic field is applied in the direction of $y$ axis through an angle $\theta=\pi / 2$. Then the equations (7) and (8) takes the form

$$
\begin{array}{ll}
\nabla^{2} V+M \frac{\partial B}{\partial x}=-1 & \text { in } \Omega \\
\nabla^{2} B+M \frac{\partial V}{\partial x}=0 & \text { in } \Omega
\end{array}
$$

Then the contours of induced magnetic field and velocity have been presented in Figure(4).

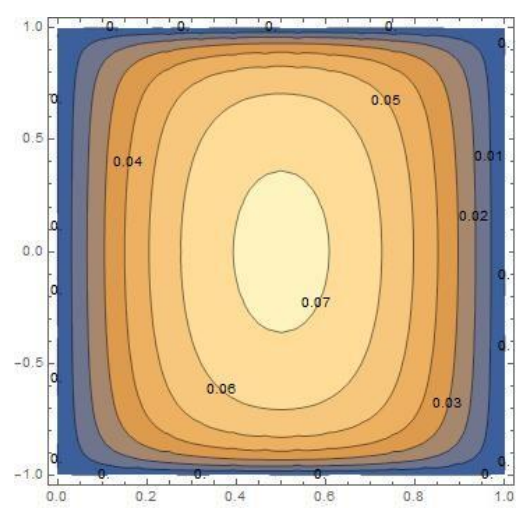

(a)

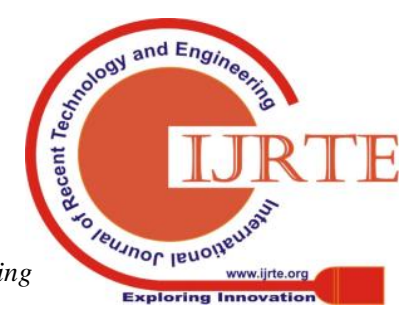




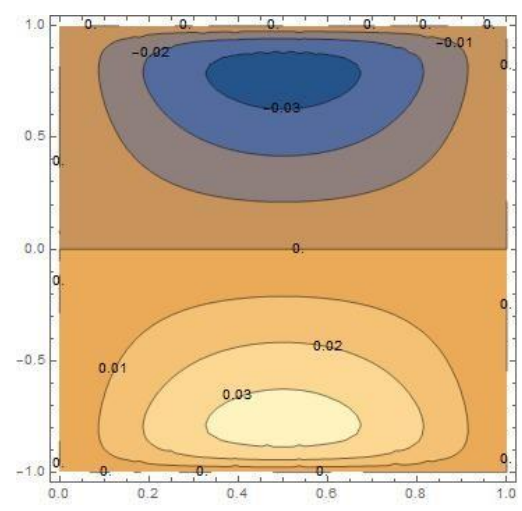

(b)

Fig. 4 Rectangular duct with $\theta=\pi / 2$ (a) Velocity

(b) Magnetic Field

\section{Triangular, Pentagon and Hexagonal Duct}

The polygon cross sections of the duct considered in this problem are triangle, pentagon and hexagon with insulated boundaries. The sides of the right angled triangle are $x=0$, $y=0$ and $x=y$. The induced magnetic field is applied in the direction of $y$ axis through an angle $\theta=\pi / 2$. On the boundary of unit circle plotting five points and six points at equal distance, pentagon and hexagon are obtained. The numerical solution of the MHD flow for the cross section of triangle, pentagon and hexagon are presented in terms of induced magnetic field and velocity contours in Figure (5 to 8).

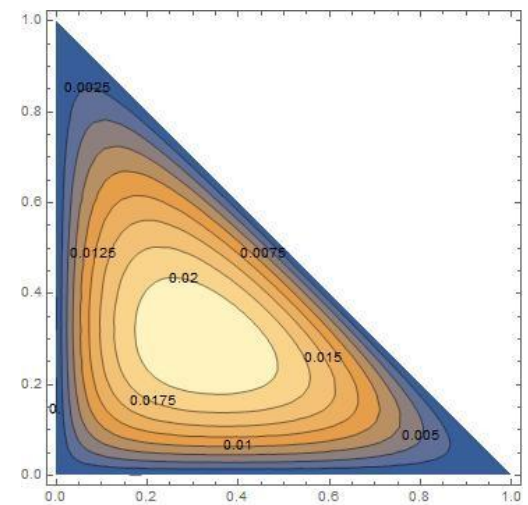

(a)

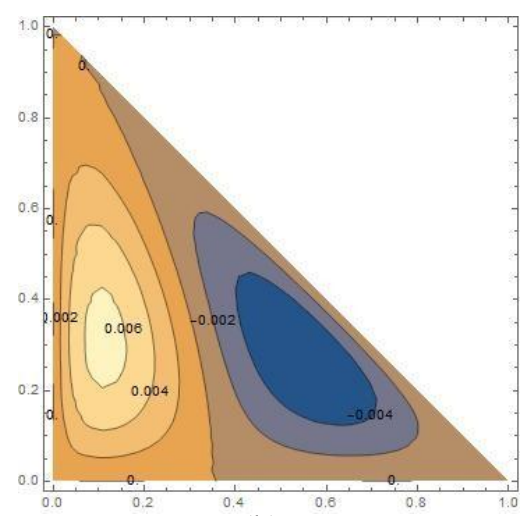

(b)

Fig.5: Triangular duct with $\theta=\pi / 2$ (a)velocity

(b) Magnetic Field

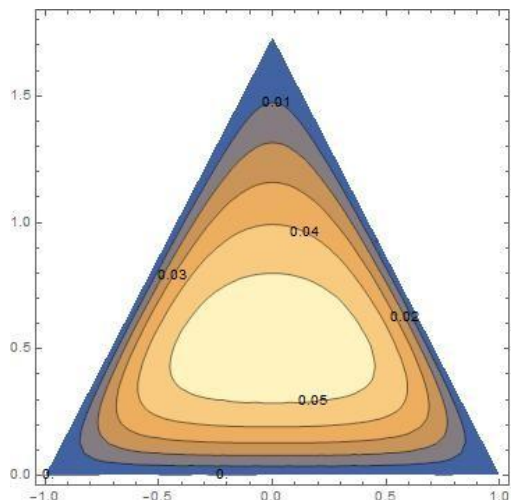

(a)

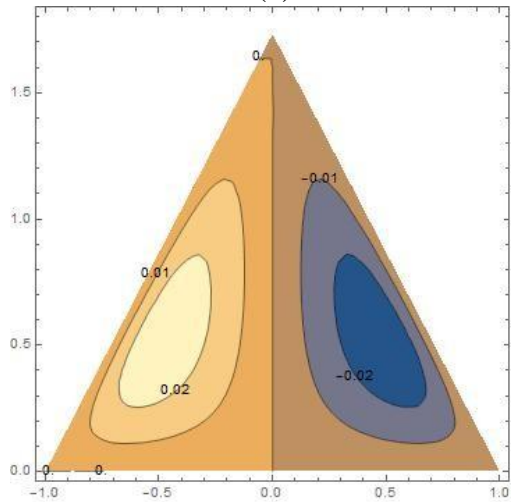

(b)

Fig.6 : Equilateral Triangle $\theta=\pi / 2$ (a) Velocity (b) Magnetic Field

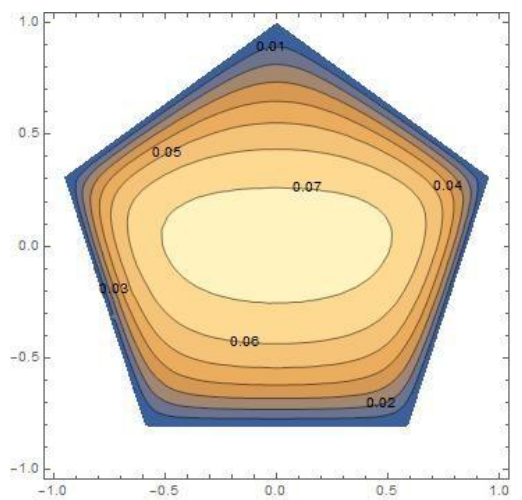

(a)

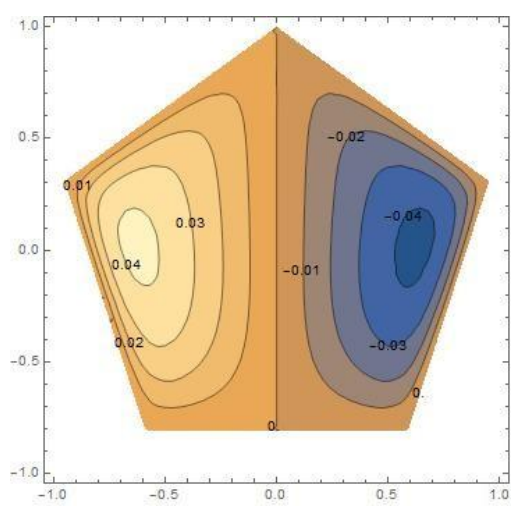

(b)

Fig.7. Pentagonal duct with $\theta=\pi / 2$ (a)velocity

(b) Magnetic Field 


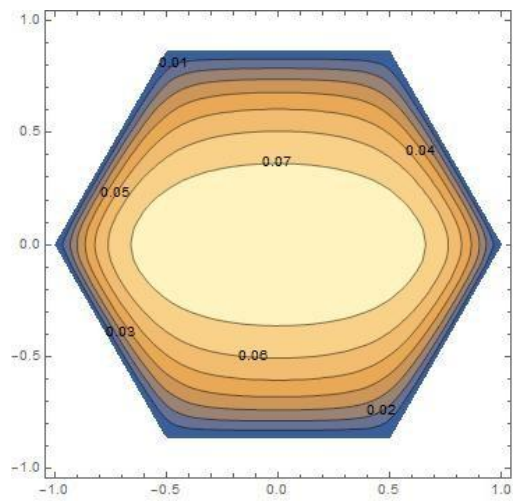

(a)

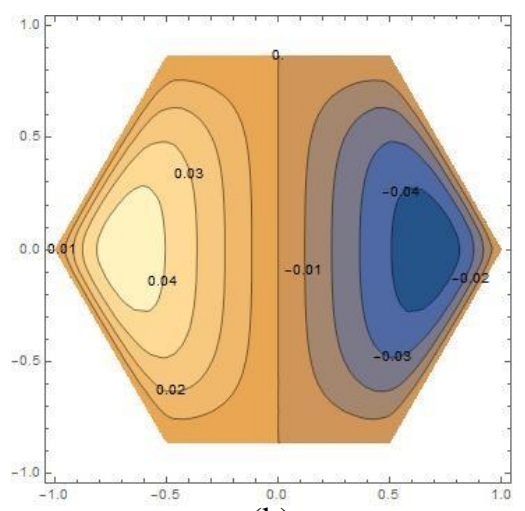

(b)

Fig. 8. Hexagonal duct with $\theta=\pi / 2$ (a) Velocity

(b) Magnetic Field

\section{CIRCULAR DUCT}

The walls of the circular duct $x^{2}+y^{2}=1$ and elliptical duct $4 x^{2}+y^{2}=4$ are electrically insulated. The incompressible electrically conducting fluid is driven in the direction of $\mathrm{z}$-axis. The magnetic field $B_{0}$ is applied in $x$ direction with $\theta=\pi / 2$. The velocity and induced magnetic field contours for circular and elliptical duct are presented in Fig. $(9,10,11)$. It is observed that the velocity profile is flat in the core of duct which match with the results presented in [3].

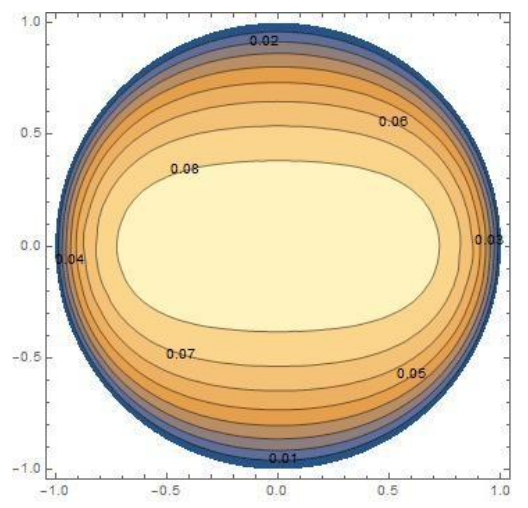

(a)

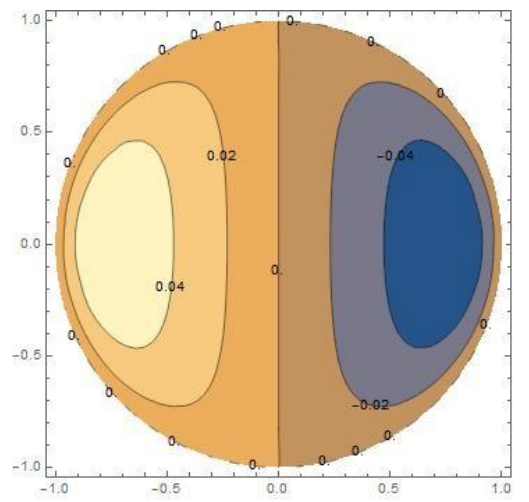

(b)

Fig. 9. Circular duct with $\theta=\pi / 2$ (a) Velocity

(b) Magnetic Field

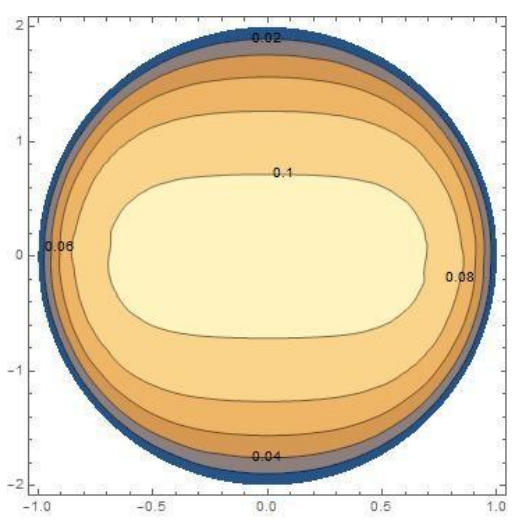

(a)

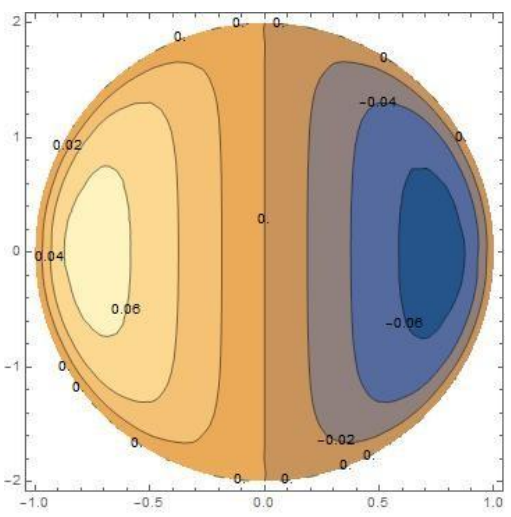

(b)

Fig. 10. Elliptical duct with $\theta=\pi / 2$ (a) Velocity

(b) Magnetic Field

\section{A. ANNULUS}

The MHD flow through the cross section of circular annulus and circular sector are considered in this problem. The boundaries of the circular annulus are defined by the region between the two circles $x^{2}+y^{2}=1$ and $x^{2}+y^{2}=1 / 4$ (i.e.) $(0 \leq \theta \leq 2 \pi$ and $0.5 \leq r \leq 1)$ are insulated. 


\section{Magnetohydrodynamic Flow Through Ducts with Different Cross Section}

The boundaries of duct with cross section as circular sector $(-\pi / 4 \leq \theta \leq \pi / 4$ and $0 \leq r \leq 1)$ are considered to study the behaviors of magnetic field and velocity.

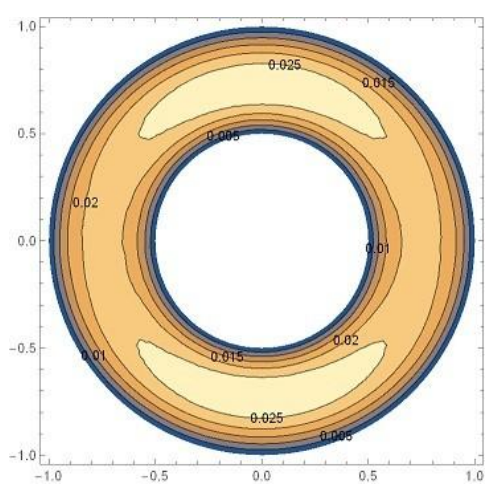

(a)

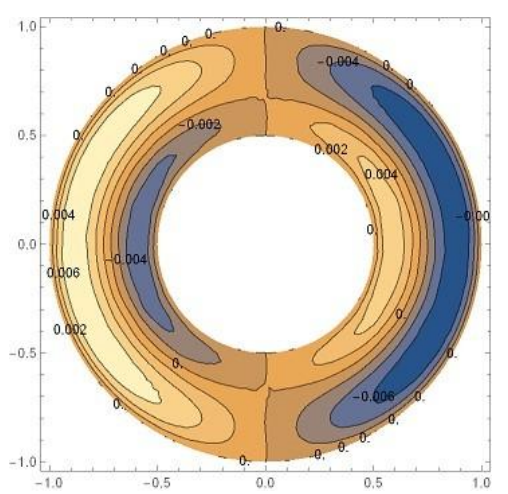

(b)

Fig. 11: Annulus duct with $\theta=\pi / 2$ (a) Velocity

(b) Magnetic Field

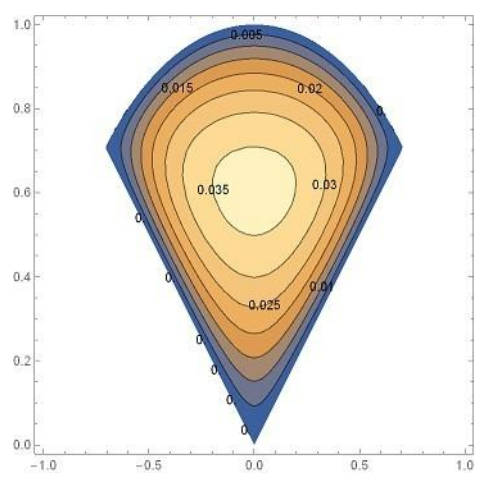

(a)

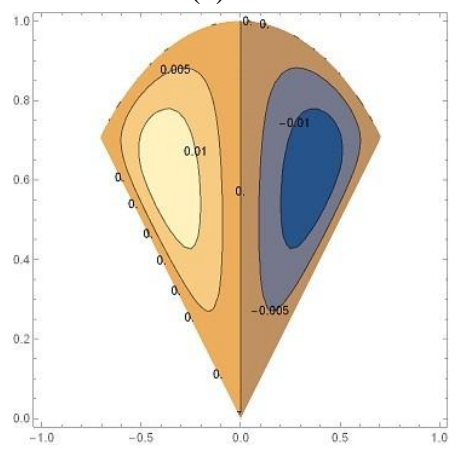

(b)
Fig. 12. Duct with sector cross section and $\theta=\pi / 2$

(a) Velocity (b) Magnetic Field

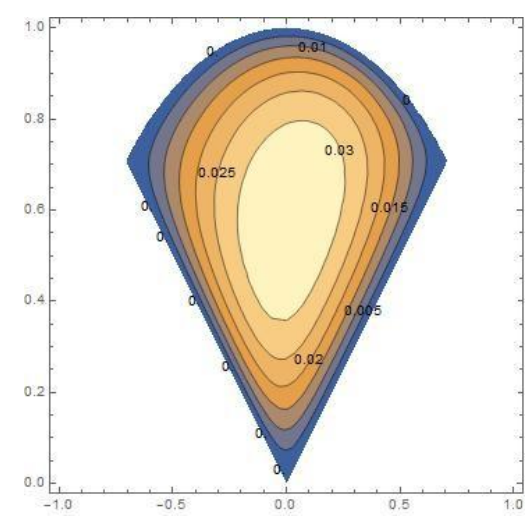

(a)

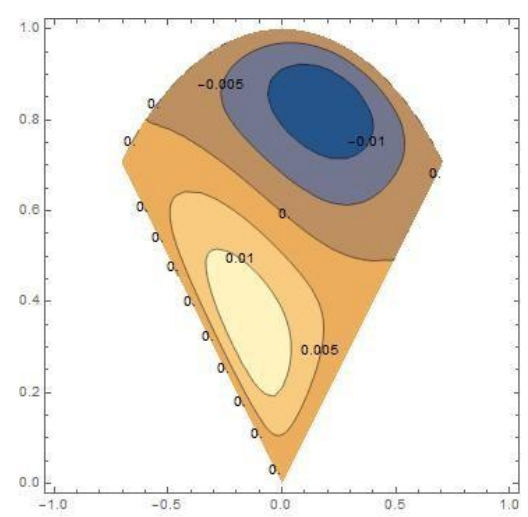

(b)

Fig. 13. Duct with sector cross section and $\theta=\pi / 6$

(a) Velocity (b) Magnetic Field

\section{CONCLUSION}

In this study, to find the solutions of MHD flow problem for different cross sections with Dirichlet boundary conditions Finite difference method have been used. The characteristics of MHD flow through channels with different cross sections are presented. To make the comparison with the shapes of the duct, Hartmann number being fixed. It is observed from the contours of MHD flow of all shapes considered that the behaviors of induced magnetic field and velocity are similar. In all the cases discussed above, velocity profile is flat in the core region which matches with behavior of velocity in the literature survey. In the future, the same problem can be solved for higher Hartmann numbers. Also, this work can be extended to Neumann and mixed boundary conditions. 


\section{REFERENCES}

1. A.I.Nesliturk and M. Tezer-Sezgin. The Finite Element Method for MHD flow at high Hartmann numbers. Computer Methods in Applied Mechanics and Engineering, 194, 1201-1224, 2005.

2. R.K.Khare Avinash. Magnetoydrodynamic flow of a dusty fluid through an equilateral triangular channel. Journal of International Academy of Physical Sciences, 17:133-144, 2013.

3. S.Han Aydin. FEM-BEM coupling for the MHD pipe flow in an exterior region. Open Journal of Fluid Dynamics, 3:184-190, 2013.

4. J. Lal B. Singh. MHD axial flow in a triangular pipe under transverse magnetic field. Indian J. Pure Appl. Math, 9:1978, 101-115.

5. J. Lal B. Singh. MHD axial flow in a triangular pipe under transverse magnetic field parallel to a side of the triangle. Indian J. Tech, 17:1979, 184-189.

6. J. Lal B. Singh. FEM in MHD channel flow problems. J. Numer. Methods Engrg., 18:11041111, 1982.

7. C.Bozkaya and M. Tezer-Sezgin. Fundamental solution for coupled Magnetohydrodynamic flow equations. Journal of Computational and Applied Mathematics, 203:125-144, 2007.

8. C.C.Chang and T.S.Lundgren. Duct flow in MHD. Angew.Math.Phy., $12,1961$.

9. Ibrahim Celikl. Solution of Magnetohydrodynamic Flow in a rectangular duct by Chebyshev collocation method. Numerical Methods in Fluids, 66:1325-1340, 2011.

10. Muhim Chutia and P. N. Deka. Numerical solution of MHD flow in a insu lated rectangular duct under the action of strong transverse magnetic field by finite difference method. International Journal of Energy and Technol-ogy, 6:1-8, 2014.

11. L. Dragosl. Magneto-fluid dynamics, England. Abacus Press, 1975.

12. Richard R. Gold. Magnetohydrodynamic pipe flow. part 1. J.Fluid Mech.,, 13:505-512, 1962.

13. J. Hartmann and F. Lazarus. Experimental investigations on the flow of mercury in a homogeneous magnetic field. Mat.Fys.Medd, 15:1-45, 1937.

14. J.A.Shercliff. Steady motion of conducting fluids in pipes under transverse magnetic fields. In Proc.Camb.Phil.Soc.,. Cambridge University, 49:136- 144, 1953.

15. J.A.Shercliff. Magnetohydrodynamic pipe flow part 2. high Hartmann number. Journal of Fluid Mechanics, 49:513-518, 1962

16. J.C.R.Hunt. Magnetohydrodynamic flow in rectangular ducts. J. of Fluid Mech, 21:577 -590, 1965.

17. M. Prasanna Jeyanthi. Numerical study of MHD flow in a rectangular duct using RBF-FD scheme. International conference on Mathematical Sciences, pages 380-384, 2014.

18. S. Kksal M. Tezer-Sezgin. Finite Element Method for solving MHD flow in a rectangular duct. Numerical methods in Engineering, 28:445-459, 1989

19. S. Han Aydn M.Tezer-Sezgin. Solution of MHD flow problems using the Boundary Element Method. Engineering Analysis with boundary elements, 30:411-418, 2006.

20. John A. Shercliffl. A textbook of Magnetohydrodynamics. Pergamon Press, 1965.

21. M. Tezer-Sezgin. BEM solution of MHD flow in a rectangular duct. $J$. Numer. Methods Fluids, 18:937-952, 1994.

22. M. Tezer-Sezgin. Solution of MHD flow in a rectangular duct by differential quardrature method. Computers and Fluids, 33:533-547, 2004.

23. Shang wu Qian and Guo qing Gu. Magnetohydrodynamic pipe flow in a duct with sector cross section. Journal of Physics A: Mathematical and General, 20(5):1087, 1987.

\section{AUTHORS PROFILE}

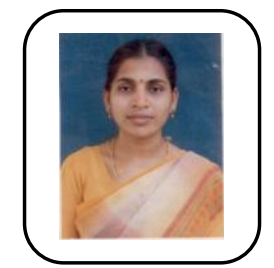

M. Prasanna Jeyanthi, working as a Assistant professor in the department of Mathematics at Sathyabama Institute of Science and Technology, Chennai. I have completed M.Sc., M.Phil in Ayya Nadar Janaki Ammal College, Sivakasi and Completed M.Tech (IMSC) in IIT Madras. I have Qualified SLET Exam. I have published 3 papers in International Conferences.

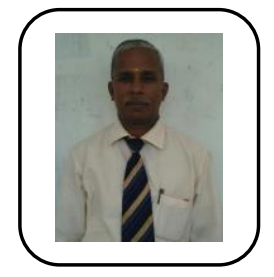

Dr.S.Ganesh, Working as professor in the department of Mathematics at Sathyabama Institute of Science and Technology. I have completed my $\mathrm{PhD}$ in Fluid Dynamics at Anna University, Chennai. I have 29 years of Teaching experience and guided $7 \mathrm{PhD}$ students and $2 \mathrm{M}$. Phil students. I have published 66 papers in National and International Journals and Conferences. I have been awarded as Best Teacher in Sathyabama twice and awarded as Best Professor in Mathematics by Business School. Affamous and dewing Menta National Education awards 2018. I have organized many National and International workshops and Conferences in Sathyabama Institute of Science and Technology. 